\title{
Genetic variability of spelt factor gene in Triticum and Aegilops species
}

\author{
Valeriya Vavilova*, Irina Konopatskaia, Alexandr Blinov, Elena Ya. Kondratenko, Yuliya V. Kruchinina and \\ Nikolay P. Goncharov
}

From Fifth International Scientific Conference "Plant Genetics, Genomics, Bioinformatics, and Biotechnology" (PlantGen2019) Novosibirsk, Russia. $24-29$ June 2019

\begin{abstract}
Background: Threshability, rachis fragility and spike shape are critical traits for the domestication and evolution of wheat, determining the crop yield and efficiency of the harvest. Spelt factor gene $Q$ controls a wide range of domestication-related traits in polyploid wheats, including those mentioned above. The main goal of the present study was to characterise the $Q$ gene for uninvestigated accessions of wheats, including four endemics, and Aegilops accessions, and to analyze the species evolution based on differences in $Q$ gene sequences.

Results: We have studied the spike morphology for 15 accessions of wheat species, including four endemics, namely Triticum macha, T. tibetanum, T. aestivum ssp. petropavlovskyi and T. spelta ssp. yunnanense, and 24 Aegilops accessions, which are donors of $B$ and $D$ genomes for polyploid wheat. The $Q-5 A, q-5 D$ and $q-55$ genes were investigated, and a novel allele of the Q-5A gene was found in accessions of T. tibetanum (KU510 and KU515). This allele was similar to the $Q$ allele of T. aestivum cv. Chinese Spring but had an insertion $161 \mathrm{bp}$ in length within exon 5 . This insertion led to a frameshift and premature stop codon formation. Thus, the T. tibetanum have spelt spikes, which is probably determined by the gene $T g$, rather than $Q$. We determined the variability within the $q-5 D$ genes among hexaploid wheat and their D genome donor Aegilops tauschii. Moreover, we studied the accessions C21-5129, KU-2074, and K1100 of Ae. tauschii ssp. strangulata, which could be involved in the origin of hexaploid wheats.

Conclusions: The variability and phylogenetic relationships of the $Q$ gene sequences studied allowed us to clarify the relationships between species of the genus Triticum and to predict the donor of the D genome among the Ae. tauschii accessions. Ae. tauschii ssp. strangulata accessions C21-5129, KU-2074 and K-1100 are the most interesting among the analysed accessions, since their partial sequence of $q-5 D$ is identical to the $q-5 D$ of $T$. aestivum cv. Chinese Spring. This result indicates that the donor is Ae. tauschii ssp. strangulata but not Ae. tauschii ssp. tauschii. Our analysis allowed us to clarify the phylogenetic relationships in the genus Triticum.
\end{abstract}

Keywords: Spike morphology, Threshability, Spelt, Rachis fragility, Q gene, Evolution, Triticum, Aegilops

\footnotetext{
*Correspondence: valeriya.vavilova@gmail.com

Institute of Cytology and Genetics SB RAS, Novosibirsk, Russian Federation
}

(C) The Author(s). 2020, corrected publication 2020. Open Access This article is licensed under a Creative Commons Attribution 4.0 International License, which permits use, sharing, adaptation, distribution and reproduction in any medium or format, as long as you give appropriate credit to the original author(s) and the source, provide a link to the Creative Commons licence, and indicate if changes were made. The images or other third party material in this article are included in the article's Creative Commons licence, unless indicated otherwise in a credit line to the material. If material is not included in the article's Creative Commons licence and your intended use is not permitted by statutory regulation or exceeds the permitted use, you will need to obtain permission directly from the copyright holder. To view a copy of this licence, visit http://creativecommons.org/ licenses/by/4.0/. The Creative Commons Public Domain Dedication waiver (http://creativecommons.org/publicdomain/zero/1. 0/) applies to the data made available in this article, unless otherwise stated in a credit line to the data. 


\section{Background}

Spike traits are critical for domestication, as they determine the crop yield and the efficiency of the harvest. At least four different loci are involved in control of spike traits in wheat species, namely, spelt factor gene $Q$ (threshability, rachis fragility and spike shape), non-brittle rachis 1 (Btr1 - spike fragility and its severity), tenacious glumes locus ( $\mathrm{Tg}$ - threshability and rachis fragility) and soft glume locus (sog - threshability) [1-9]. $Q$ gene located on the long arm of the chromosome $5 \mathrm{~A}$ controls a wide range of domestication-related traits in polyploid wheat. Molecular cloning allowed the $Q$ gene to be referred to the APETALA2 (AP2)-like transcription factors and allowed two functional alleles $Q-5 A$ and $q-5 A$ to be described [1].

Most of the analysed cultivated wheat species are characterized by free-threshing, normal (or compact) spikes with non-fragile rachis and the presence of allele Q-5A. In contrast, the allele $q-5 A$ has been described in wild wheats with non free-threshing fragile spelt spikes $[1,2$, 10]. Two single-nucleotide polymorphisms (SNPs) were described for the $Q$ and $q$ alleles: (1) $G$ to $C$ transition within exon 8 close to the AP2 domain regions, which results in non-synonymous substitution from valine to isoleucine and (2) neutral $\mathrm{C}$ to $\mathrm{T}$ substitution in the miRNA172 binding site within exon 10 [1-4, 11].

The regulatory mechanisms of $Q$ gene expression remain poorly investigated. Based on the results of a twohybrid yeast analysis, Simons et al. (2006) suggested that the presence of isoleucine at the 329 position, led to effective homodimer formation, which increases the Q-5A expression (1). On the other hand, it was shown that plant miRNA172 plays important role in floral development and regulation of $A P 2$-like transcription factors [10, 12-15]. In recent studies, a great deal of attention has been paid to the role of miRNA172 in the regulation of the $Q$ gene, since the variation in the miRNA172 target site increases the $Q$ expression [16-18]. Thus, in mutants of the bread wheat cultivar Sunstate, Greenwood et al. (2017) described the allele Q', which possesses the additional SNP within the miRNA172 binding site. The mutant plants showed a reduced height, compact spike phenotype and higher expression of the $Q$ ' allele (compared to the $Q$ allele), due to the reduced level of miRNA172-dependent mRNA degradation, since miRNA172 cleaves the $q$ allele transcripts more efficiently than those of $Q$ and the reduced activity of miRNA172 causes spike compactness and easier threshing. Moreover, it was shown that overexpression of miRNA172 in transgenic wheats led to the formation of non-free-threshing (naked) grains and elongated spikes with an increased number of florets per spike [16]. Liu et al. (2018) showed that the $Q$ gene is a nuclear transcriptional repressor which interacts with co-repressor
TOPLESS, and they suggested that in Triticum aestivum L., $Q$ expression is regulated by both miRNA172 and TOPLESS.

Despite the fact that $q-5 B$ represents a pseudogene and $q-5 D$ is expressed at a lower level compared to $Q$ $5 A$, they both contribute to the suppression of spelt spike formation [2]. Recently a new allele $q^{\prime}-5 D$ with a point mutation within the miRNA172 binding site, was identified [19]. The allele is characterized by an increased transcription level and pleiotropic effects on the spike compactness and plant dwarfness in T. aestivum cv. NAUH164. However, the $Q$ gene is poorly investigated in Aegilops species, which are considered to be the donors of $\mathrm{B}$ and $\mathrm{D}$ genomes for polyploid wheat.

In the present study, $Q$ genes were investigated from accessions of wheat species (including four endemic species, namely, T. macha Decapr. et Menabde, T. tibetanum Shao, T. aestivum ssp. petropavlovskyi (Udacz. et Migusch.) N.P. Gontsch. and T. spelta ssp. yunnanense (King ex S.L. Chen) N.P. Gontsch.), Aegilops speltoides Tausch and Ae. tauschii Coss. Based on variability and the phylogenetic relationships of $Q$ gene sequences, we suggest the scheme of Triticum L. and Aegilops L. evolution.

\section{Results}

\section{$Q$ gene alleles in genus Triticum species}

In the present study, we determined three spike morphology traits for 15 accessions of wheat species including diploids (T. monococcum L., T. urartu Thum. ex Gandil., T. boeoticum Boiss.) and endemic hexaploids ( $T$. macha Decapr. et Menabde, T. aestivum ssp. petropavlovskyi (Udacz. et Migusch.) N.P. Gontsch., T. spelta ssp. yunnanense (King ex S.L. Chen) N.P. Gontsch., T. vavilovii (Thum.) Jakibz., T. tibetanum Shao) (Fig. 1, Additional file 1: Table S1).

We amplified full-length sequences of the $Q-5 A$ gene in three diploid and four hexaploid wheat species. The obtained sequences were compared with known $Q$ and $q$ alleles of wheat species available from GenBank. Comparative analysis and the following phylogenetic analysis showed that all diploid accessions and T. macha accessions with non free-threshing fragile spelt spikes had allele $5 A q$, identical to $T$. dicoccum (AY714343) (Fig. 2).

Sequences of Q-5A gene from two accessions of $T$. aestivum ssp. petropavlovskyi with free-threshing non fragile spelt-like spikes were identical to the allele Q-5A from $T$. aestivum cv. Renan (JF701614). The allele $q$ of T. spelta (AY714341) was determined in accessions of $T$. spelta ssp. yunnanense, which were characterized by non free-threshing non fragile spelt spikes.

A novel allele of the Q-5A gene was described for $T$. tibetanum (KU510 and KU515) (Fig. 2, Additional file 1: Table S1). The allele was similar to the $Q$ allele of $T$. 

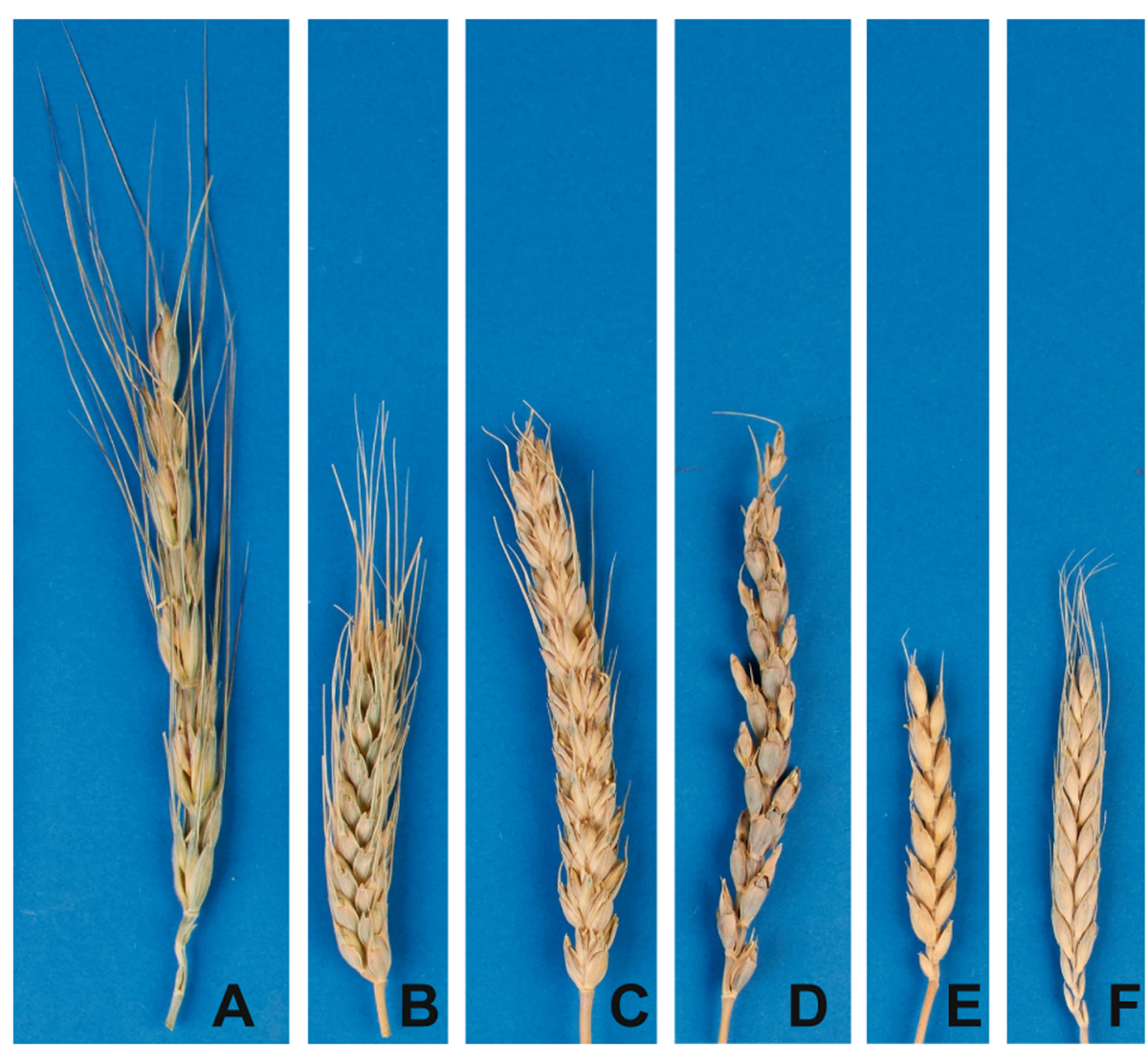

Fig. 1 Spike morphologies of wheat species. a T. aestivum ssp. petropavlovskyi K-43351 (free-threshing, non fragile, spelt-like). b T. spelta ssp. yunnanense KU506 (non free-threshing, non fragile, spelt). c T. spelta ssp. yunnanense KU509 (non free-threshing, non fragile, spelt). d T. vavilovii Tri4630 (non free-threshing, non fragile, spelt). e T. tibetanum KU510 (non free-threshing, fragile (second type), spelt). f T. macha K-31689 (non free-threshing, fragile (second type), spelt)

aestivum cv. Chinese Spring, excluding an insertion $161 \mathrm{bp}$ in length within exon 5 (Fig. 2). This insertion led to a frameshift and premature stop codon formation. In parallel, Jiang et al. (2019) described this allele for accessions of $T$. tibetanum and designated it $Q^{t}$. The transposon insertion has led to re-acquisition of a wild trait (brittle rachises) in T. tibetanum [20]. No miRNA binding sites were predicted for the insertion region by psRNATarget.

We did not find the allele $Q^{\prime}-5 A$ previously described for the T. aestivum cv. Sunstate mutant, among the analysed accessions [17].

We obtained no positive results from the PCR amplifications of Q-5A in the analysed accessions of $T$. vavilovii (Additional file 1: Table S1). The primers used in the present study allowed us to amplify only the full-length $q-5 D$ gene of this species. Comparative and phylogenetic analysis confirmed that the studied $T$. vavilovii accessions possess an allele of the $q-5 D$ gene identical to that of $T$. aestivum $\mathrm{cv}$. Chinese Spring (Additional file 1: Table S3).

T. macha, T. aestivum ssp. petropavlovskyi, T. spelta ssp. yunnanense, and T. tibetanum, are characterized by the presence of allele $q-5 D$, while the $q^{\prime}-5 D$ allele was not found in any of the analysed wheat species (Fig. 2, Additional file 1: Table S1). Comparison of the $q-5 D$ gene with those of $T$. aestivum revealed species-specific and accession-specific substitutions within the introns (Additional file 1: Table S3). Two accessions possess the substitutions within the 10th exon. The Q-5D gene of $T$. aestivum ssp. petropavlovskyi is characterized by two non-synonymous substitutions, leading to the 397Ala- > $\mathrm{Val}$ and 405Ser->Pro mutations. The synonymous substitution is presented in the position $3098(\mathrm{G}->\mathrm{T})$ of the $Q-5 D$ of T. vavilovii (Additional file 1: Table S3).

\section{$Q$ gene alleles in Ae. tauschii and Ae. speltoides}

In the present study, we determined the spike morphology traits for 24 accessions of Aegilops species, which are donors of $\mathrm{B}$ and $\mathrm{D}$ genomes for polyploid wheat (Additional file 1: Table S4). Analysed accessions of Ae. tauschii were characterized by one of the following phenotypes: non-free-threshing fragile spelt or free-threshing fragile spelt (mutant accession TQ27) (Additional file 1: Table S4). 


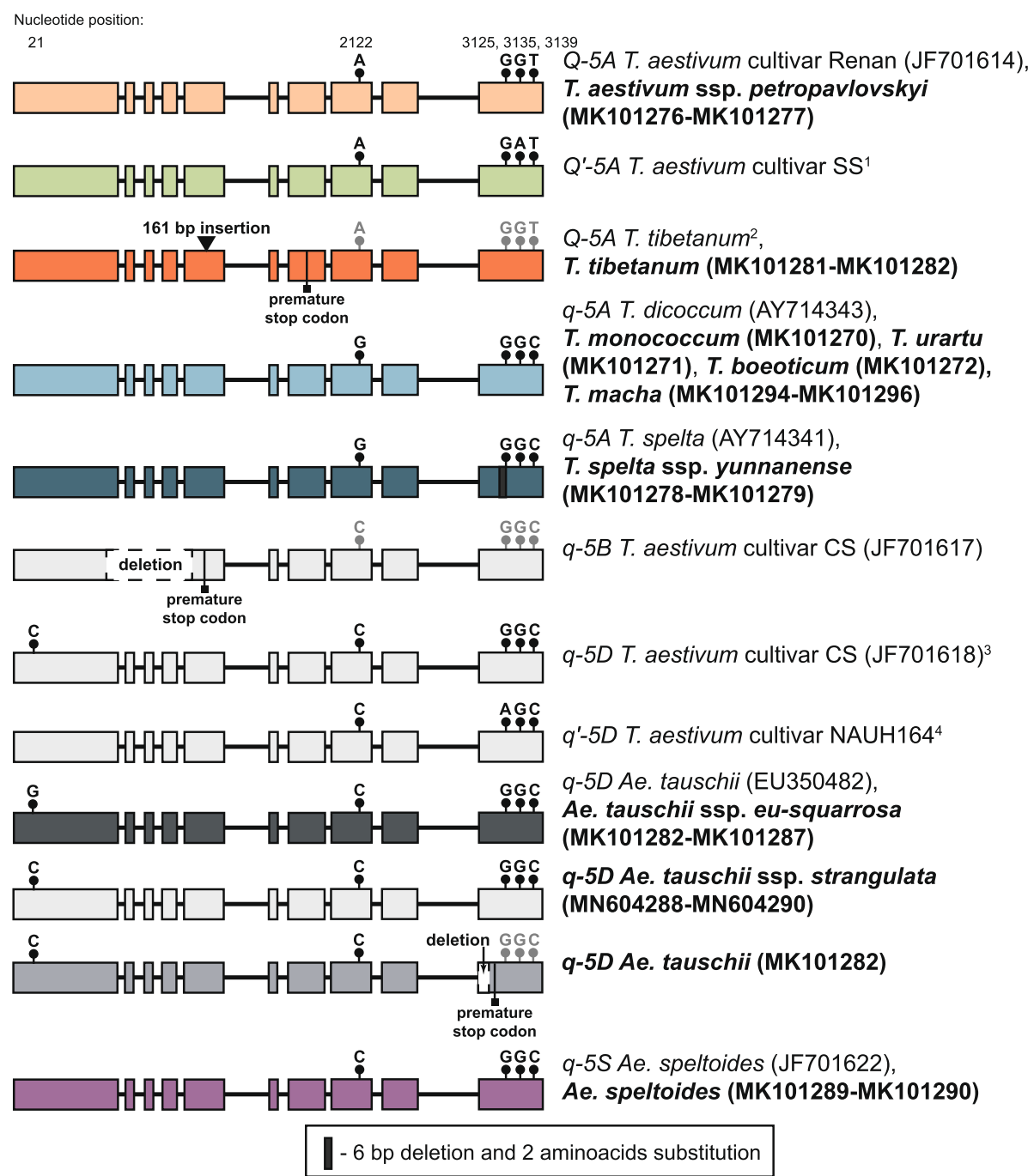

Fig. 2 Scheme of $Q$ gene alleles variability from various Triticum and Aegilops species. The numbers of nucleotides upstream from the start codon are given in accordance with the sequence Q-5A for T. aestivum (JF701619). Different A genome alleles are marked by different colours. Insertion is indicated by a black triangle. Single nucleotide polymorphisms are indicated by black circles. [1]- The Q'-5A allele was described by Greenwood et al. (2017); [2]- the $Q^{t}$ allele was described in the present study in parallel with the study by Jiang et al. (2019); [3]- and T. macha (MK101294MK101296), T. tibetanum (MK101301-MK101302), T. spelta ssp. yunnanense (MK101299-MK101230), T. vavilovii (MK101291-MK101293), T. aestivum ssp. petropavlovskyi (MK101297-MK101298); [4]- The q'-5D allele was described by Zhao et al. (2018)

Full-length $q-5 D$ gene sequences were obtained for ten Ae. tauschii accessions (MK101282-MK101288, MN604288-MN604290). These sequences were compared with known $q-5 D$ sequences from Ae. tauschii and hexaploid wheat, including those identified in the present study. The sequences of the $q-5 D$ gene's exons are highly conservative and the differences between the analysed sequences are limited to the introns. However, we have described a new allele with an SNP in position 72 and a 12-bp deletion affecting intron 9 and exon 10 in Ae. tauschii KU2001 (Fig. 2). The substitution G->A is synonymous, but the deletion causes a frameshift and the potential formation of a defective protein (Fig. 2).
Several newly obtained sequences from Ae. tauschii accessions differed from allele $q-5 D$ of Ae. tauschii EU350482 and were closer to the D-genome sequences from hexaploids (Additional file 1: Table S3). The $Q$ gene of the accessions $\mathrm{K}-1216$ and TQ27 was similar to the $q-5 D$ of hexaploids, not only in substitutions within introns but also with respect to the G-> C transition in position 21 (Fig. 2).

Three accessions of Ae. tauschii (C21-5141, C215129, and KU-2074) are the most interesting among the analysed accessions, since their sequences of $q-5 D$ are highly similar or even identical in case of C21-5129 and KU-2074 to the $q-5 D$ of T. aestivum cv. Chinese Spring (JF701618) and T. aestivum cv. Renan (JF701615) 
(Additional file 1: Table S3). Furthermore, we analysed the most variable region of $q-5 D$, including intron 9 , exon 10 and partial 3'UTR, in 10 additional accessions of Ae. tauschii (Additional file 1: Table S1). Similarly to three accessions described above, the partial sequence of $q-5 D$ from Ae tauschii $\mathrm{K}-1100$ is identical to the $q-5 D$ of accessions of polyploid wheat (Additional file 1: Table S3). Moreover, the 3'UTR of the three novel sequences (MN206106, MN604289, MN604290) possess a CTtrack of identical length to that described for the two well-studied cultivars of $T$. aestivum, directly confirming the participation of Ae. tauschii ssp. strangulata in the formation of hexaploid wheats.

Full-length $q-5 S$ gene sequences were obtained for four Ae. speltoides accessions (Fig. 2, Additional file 1: Table S4). The comparative analysis revealed that they are quite different from the $q-5 B$ sequences of polyploid wheats.

\section{Phylogeny of the $Q$ genes sequences}

Phylogenetic analysis was performed for full-length DNA sequences of newly obtained and known $Q$ gene sequences of different wheat species. In total, 70 sequences of $\mathrm{A}, \mathrm{B}, \mathrm{D}$ and $\mathrm{S}$ genome copies of the $Q$ gene were used for the construction of a phylogenetic tree (Fig. 3). Three major clusters are present on the resultant tree. Cluster I consists of the $Q$ gene sequences of the B genome of Triticum species and of the $\mathrm{S}$ genome of Ae. speltoides, where the latter form a separate group.

The D genome copies of the $Q$ gene are included in two subclusters of cluster II. The Ae. tauschii species are present in both subclusters, i.e., the five sequences identified in the present study are clustered together with $q$ $5 D$ of the $T$. aestivum and $T$. vavilovii accessions (Fig. 3).

The third cluster has a clearly identifiable subcluster with $q-5 A$ sequences of the diploids $T$. sinskajae, $T$. boeoticum and T. monococcum. The remaining part of cluster III has a structure which is less clearly defined and is formed by both $q-5 A$ and $Q-5 A$ alleles from $T$. urartu and polyploid wheat species. Nevertheless, Q-5A sequences of T. tibetanum and T. aestivum ssp. petropavlovskyi were grouped together with tetraploid and hexaploid wheat species that possess the Q-5A allele. Within the cluster III, a branch with high support can be identified. This branch includes T. tibetanum, T. aestivum ssp. petropavlovskyi, T. aestivum, T. polonicum, T. compactum and T. sphaerococcum.

$Q$ gene sequences of the Chinese endemic species $T$. spelta ssp. yunnanense, are located along with European $T$. spelta, which may confirm human participation in creating this species. The $T$. macha sequences form a separate branch within the group of $q$-5A-allele sequences. Sequences of $T$. urartu are located separately from polyploid wheats but do not form a single subcluster.

\section{Discussion}

The origin of modern cultivated polyploid wheat species is a complicated and not fully elucidated history, which includes the emergence of species of the genus Triticum in both a natural way and as a result of human activity. Using a combination of bioinformatics and experimental methods of research usually allows the phylogenetic relationships of species within different taxa to be established. Unfortunately, species of the genus Triticum appeared about 10,000 years ago, and all the molecular markers used have a minimum number of nucleotide substitutions, which does not allow the phylogenetic relationships of all known species of this genus to be established in full. The standard chloroplast and mitochondrial markers allow the phylogeny of the genomes A, B, D and $\mathrm{G}$ to be determined, but do not allow the phylogenetic relationships of these species to be revealed [21, 22].

Therefore, an approach based on the analysis of various mutations occurring in the genes encoding the traits involved in domestication, most of which are transcription factors, was quite fruitful for identifying the emergence of species of the genus Triticum and establishing their phylogenetic relationships. This approach has been used in several studies, with a view to elucidating the evolution of diploid and tetraploid wheat species. In the present work, we used differences in the Q-5A and Q-5D genes to clarify the phylogeny of the genera Triticum and Aegilops.

It is known that the source of the A genome for all hexaploid species was $T$. urartu, and the source of both $\mathrm{B}$ and $\mathrm{G}$ genomes was Ae. speltoides, whose $\mathrm{S}$ genome is the ancestor for the B and G genomes in wheat. In all diploid wheat species, including the mutant $T$. sinskajae, only the variant VIGGC (329 valine and GGC motif within the miRNA172 binding site in the 10th exon) has been detected so far [present study; 1]. However, since accessions with fragile rachises and non fragile rachises are represented among diploid wheat species, the gene Btr1-A attracted attention of researchers [5, 6]. Pourkheirandish et al. (2018) identified that the non-synonymous change at the coding region of Btr1-A (A119T) determines the rachis fragility trait in diploid einkorn wheat species. The relationship between the Btr1 and Q genes, as well as their joint contribution to the rachis fragility are poorly understood.

All hexaploids received the $\mathrm{D}$ genome from $A e$. tauschii, and several studies have emphasized that the donor is Ae. tauschii ssp. strangulata (Eig) Zvelev but not Ae. tauschii ssp. tauschii [23-26]. In the present study, we established that several allelic variants of $q-5 D$ are present in the Ae tauschii accessions. We have 


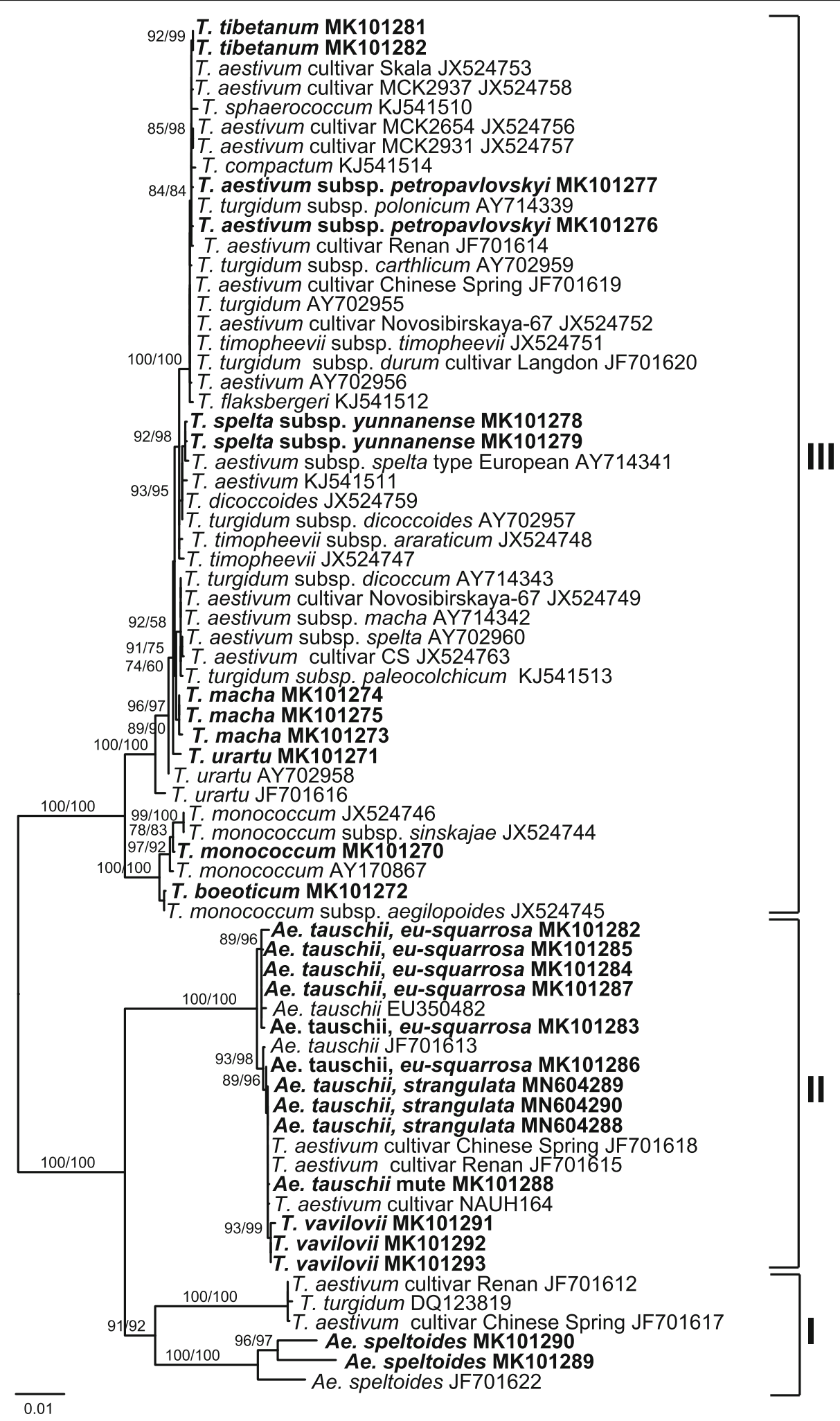

Fig. 3 Maximum likelihood tree constructed on the basis of the full-length $Q$ gene sequences from various Triticum and Aegilops species. Statistical support for the ML phylogeny was evaluated by ultra-fast bootstrap/aLRT. Nodes with statistical support over $50 \%$ are indicated. Clades are highlighted in different colours. Sequences identified in the present study are marked in bold 
identified $q-5 D$ sequences from Ae. tauschii accessions (C21-5129, KU-2074, and K-1100) that are highly similar to those of hexaploid wheat accessions. The accession $\mathrm{K}-1100$ is originated from Azerbaijan and the accessions C21-5129 and KU-2074 are originated from Iran. According to modern data, the crossing between the wild tetraploid wheat progenitor and Ae. tauschii, which led to the emergence of hexaploid wheat, occurred about 7000-7500 years ago in Central Asia [27]. This allows us to suggest that the accessions C21-5129, KU-2074, and K-1100 of Ae. tauschii ssp. strangulata could have been involved in the origin of hexaploid wheat species.

The $Q$ gene is represented in the A genomes of hexaploids in three variants: V\GGC, I\GGT (329 isoleucine and GGT motif within the miRNA172 binding site in the 10th exon) and IIGAT (329 isoleucine and GAT motif within the miRNA172 binding site in the 10th exon). The original V\GGC variant of the species $T$. macha and T. spelta ssp. indo-europeum Vav. was directly obtained from tetraploid $T$. dicoccum and $T$. spelta ssp. irano-asiaticum Flaksb., which probably acquired it from the original tetraploid ancestor. T. spelta ssp. yunnanense stands apart, since this species carries a unique deletion in the $Q$ sequence, which was found only in European T. spelta (AY714341). It should be noted that all the above-mentioned species contain the wild-type $Q$ gene. Moreover, the Georgian endemic T. macha is located on the scheme separately from $T$. spelta ssp. indo-europeum and T. spelta ssp. yunnanense, due to unique mutations within the $q-5 D$ gene.

The remaining hexaploid species, including $T$. aestivum, have the IIGGT variant of the $Q$ gene in the A genome, which does not originate from $T$. dicoccoides. It should be noted that another unique variant of the $Q$ gene, I $\backslash$ GAT, was found in the genome of $T$. aestivum cv. Chinese Spring. As for T. sphaerococcum and $T$. compactum, T. aestivum ssp. petropavlovskyi probably originated from T. aestivum.

The results of Jiang et al. (2019) showed that the unique Q-5A allele of $T$. tibetanum originated from the $Q$ gene of T. aestivum. The fragile rachis of T. tibetanum is a result of the re-acquisition of wild traits that occurred during dedomestication. According to our investigations, the $q-5 D$ gene sequences have two substitutions, which are described for the T. macha accessions only. The fragile rachis of the $T$. aestivum and the spelt spikes of T. tibetanum are probably determined by the gene $T g$, and not by $Q[9,28]$. $T g$ was described for hexaploid wheat species and localized on the short arm of the chromosome 2D, but until this gene has been molecularly cloned it is impossible to identify it's relation to the gene $Q$.

Thus, our analysis allowed us to clarify the phylogenetic relationships of species of the genus Triticum (Fig. 4).

\section{Conclusions}

The origin of cultivated wheat species and traits essential for domestication still remain an open question. Investigation of the genes which determine the traits involved in domestication of wheat allow to describe new alleles and are also promising for establishing the history of wheat's origin. In this study, we studied the spelt factor gene, $Q$, for 15 accessions of wheat species, including four endemics, and 24 Aegilops accessions. Our analysis allowed us to clarify the phylogenetic relationships in the genus Triticum, namely show that the donor of the $\mathrm{D}$ genome for species of the genus Triticum is Ae. tauschii ssp. strangulate.

\section{Methods}

\section{Plant material and growth conditions}

Germplasm of di- and hexaploid wheat and Aegilops species, were obtained from 10 gene banks (Additional file 1: Table S1, Table S4). For each accession, 10 plants were grown under standard greenhouse conditions.

Threshability and rachis fragility traits were assessed using manual threshing. Spikes were classified as free threshing if their grains were surrounded by soft glumes which came off during threshing. Spikes with grains covered by tough glumes remaining adhered to the grain after the threshing were determined as non-free threshing. Non-fragile spikes were those which did not undergo self-dependent disarticulation, while fragile spikes displayed W- or B-type disarticulation. Spikes which separated from the whole straw without breaking into spikelets were a second type of fragile spike [29].

Spike shape has been determined visually in accordance with the following definitions: (1) the pyramidal spike with an elongated rachis and tenacious glumes is the spelt spike; (2) the short squareheaded parallel-sided spike is the normal spike; (3) the elongated spindleshaped spike is the spelt-like spike. The spike shape has been confirmed by calculation of Flaksberger's formula for spike density, $\mathrm{D}=10(\mathrm{~A}-1) / \mathrm{B}$, where $(\mathrm{A}-1)$ is the number of spikelets per spike excluding the apical spikelet, and $B$ is the length of the spike rachis in $\mathrm{cm}$ [30]. Calculated values for $\mathrm{D}$ displayed a high correlation with visual assessment of spike shape (see [31]).

\section{Total DNA extraction, PCR amplification, cloning and sequencing}

Total DNA was isolated from $100 \mathrm{mg}$ of leaves using the DNeasy Plant Mini Kit (QIAGEN) according to the manufacturer's protocol. The $Q-5 A$ gene sequences of Triticum species and the Q-5D gene sequences of Aegilops species were PCR-amplified as six separate overlapping fragments, using primer pairs and PCR conditions as previously published [11]. In order to identify SNP (G->A) within the miRNA172 binding site the partial 


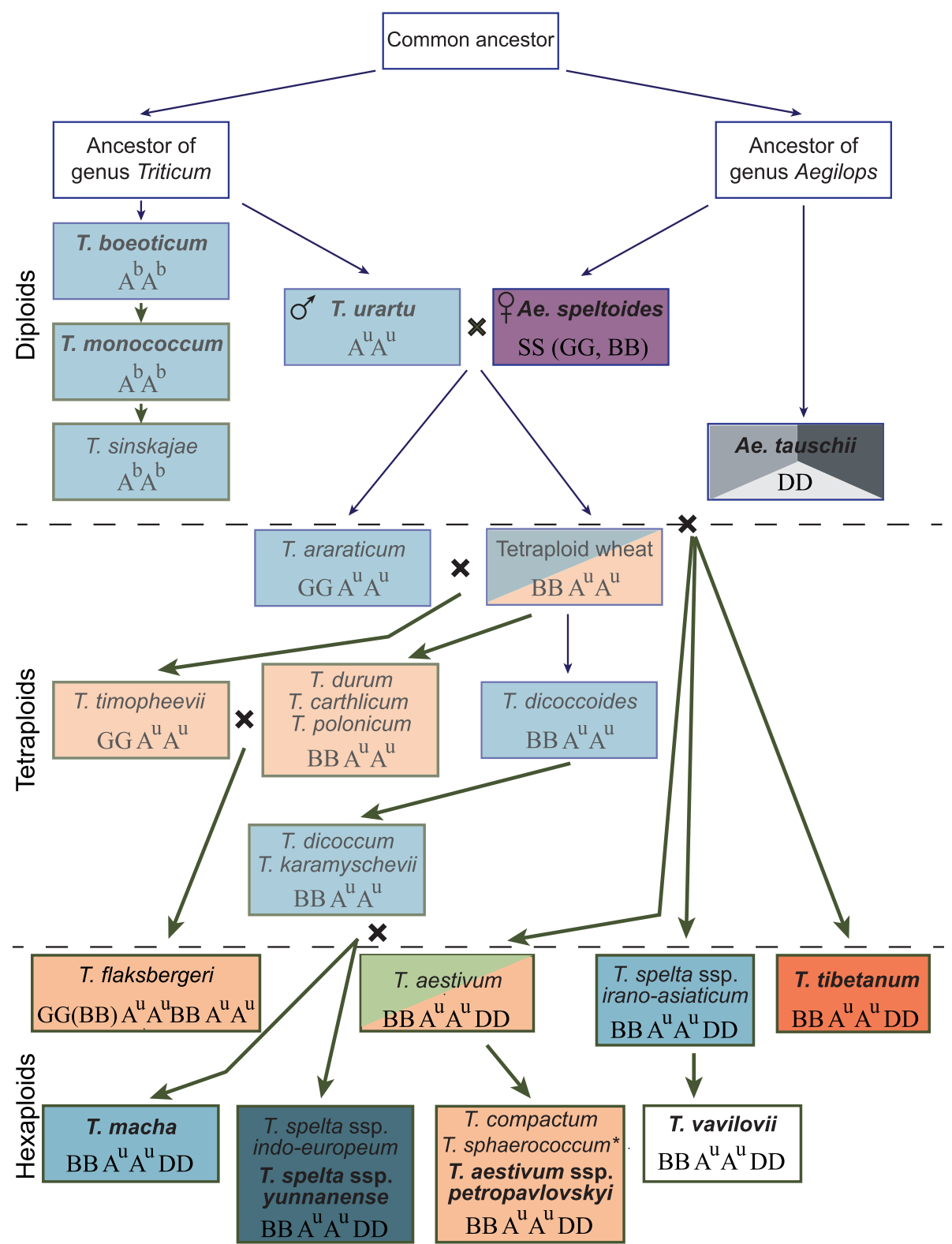

Fig. 4 Predicted evolution of Triticum and Aegilops genera. Boxes are coloured according to the $Q$ gene alleles detected in the accessions of the species (box colours match the colours in Fig. 2). Ancestral species are shown in white boxes. Blue arrows indicate natural selection and green arrows indicate artificial selection (domestication). Indicates the Q gene allele with isoleucine in the 329 position and SNPs 3125G, 3135G and 3139C. T. karamyschevii = T. turgidum ssp. paleocolchicum

sequences of the $q-5 D$ gene covering the region from intron 6 to exon 10 were amplified for hexaploid wheat accessions using the primer pair (Q-D1F, Q-D5R) designed by Zhao et al. [19]. PCR products were separated by agarose gel electrophoresis and purified using a QIAquick Gel Extraction Kit (QIAGEN). Purified PCR fragments were cloned into a $\mathrm{pGEM}^{\circ}$-T Easy vector using a pGEM-T Easy kit (Promega) and amplified using M13 primers prior to sequencing. Sequencing reactions were performed using $200 \mathrm{ng}$ of the PCR product and a BigDye Terminator Kit on an ABI 3130xl Genetic
Analyzer (Applied Biosystems) at the SB RAS Genomics Core Facility (http://www.niboch.nsc.ru/doku.php/core facility). In total, 10 clones were sequenced for each fragment of Q-5A and Q-5D gene from all the studied accessions. $Q$ gene sequences were deposited in GenBank.

\section{Sequence analysis}

Multiple sequence alignment was performed using the MAFFT algorithm v. 7.397 [32] and then analysed in AliView 1.18.1 [33]. The p-distance was calculated using the Jukes-Cantor model in MEGA v. 5.10 [34]. A $Q$ gene 
sequence region from the start codon to the stop codon (including introns) was used for phylogenetic analysis using the Maximum likelihood method in IQ-TREE v. 1.6.5 [35]. The best-fit evolutionary model $(\mathrm{HKY}+\mathrm{F}+\mathrm{I})$ for the analysis according to the Bayesian information criterion (BIC), was evaluated using the ModelFinder algorithm implemented in the IQ-TREE package. Ultrafast bootstrap and aLRT were used to estimate the statistical credibility of the obtained phylogenetic clades [36]. A $Q$ gene sequences search was performed using BlastN and BlastP at https://www.ncbi.nlm.nih.gov/. OFR was predicted using the ORFfinder program, which was used for the search for open reading frames (ORFs) in the DNA sequence (https://www.ncbi.nlm.nih.gov/orffinder/ ). The miRNA binding sites were identified using the psRNATarget server [37].

\section{Supplementary information}

Supplementary information accompanies this paper at https://doi.org/10. 1186/s12870-020-02536-8.

Additional file 1: Table S1. Wheat species used in the study, their spike morphologies and Q gene alleles. Table S2. Spike shape determination for the wheat species used in the present study. Table S3. Variability of the $q-5 D$ gene sequences among Aegilops and Triticum species. Table S4. Aegilops species used in the study, their spike morphologies and $Q$ gene alleles.

\section{Abbreviations}

BIC: Bayesian information criterion; ORFs: Open reading frames; PCR: Polymerase chain reaction; RNA: Ribonucleic acid; SNPs: Singlenucleotide polymorphisms; UTR: Untranslated region

\section{Acknowledgements}

We thank A. E. Kuznetsova, S. Thumanyan and S. Koval (Institute of Cytology and Genetics SB RAS) for assistance in preparation of photographic images.

\section{About this supplement \\ This article has been published as part of BMC Plant Biology Volume 20 Supplement 1, 2020: Selected articles from the 5th International Scientific Conference "Plant genetics, genomics, bioinformatics, and biotechnology" (PlantGen2019). The full contents of the supplement are available online at https://bmcplantbiol.biomedcentral.com/articles/supplements/volume-20- supplement-1.}

\section{Authors' contributions}

NPG designed the study and performed the greenhouse experiments. EYK and YVK performed the genetic experiments and analysed the results. $W$ and IK performed the molecular studies. W and $A B$ analysed the $Q$ gene sequences. IK and $\mathrm{W}$ wrote the manuscript. $\mathrm{AB}$ and NPG reviewed the manuscript. All authors read and approved the final manuscript.

\section{Funding}

Funding for this work was provided by the Russian Science Foundation (grant number: 16-16-10021). Publication of this article has been funded by the Russian Science Foundation (grant number: 16-16-10021). The Russian Science Foundation (grant number: 16-16-10021) has supported the design of this study and the data collection, analysis, and interpretation and manuscript writing.

\section{Availability of data and materials}

The $Q$ gene sequences are available in GenBank, with the accession numbers: MK101270-MK101302, MK101282-MK101290, MN206102-MN206113, MN604288-MN604290. Raw phylogenetic data were deposited at the
FigShare (https://doi.org/10.6084/m9.figshare.12562148). The datasets used and/or analysed during the current study available from the corresponding author on reasonable request.

Ethics approval and consent to participate

Not applicable.

\section{Consent for publication}

Not applicable.

\section{Competing interests}

The authors declare that they have no competing interests.

Received: 10 September 2019 Accepted: 29 June 2020

Published online: 14 October 2020

\section{References}

1. Simons KJ, Fellers JP, Trick HN, Zhang Z, Tai YS, Gill BS, et al. Molecular characterization of the major wheat domestication gene Q. Genetics. 2006; 172:547-55.

2. Zhang Z, Belcram H, Gornicki P, Charles M, Just J, Huneau C, et al. Duplication and partitioning in evolution and function of homoeologous $Q$ loci governing domestication characters in polyploid wheat. Proc Natl Acad Sci. 2011;108:18737-42.

3. Muramatsu M. Dosage effect of the spelta gene $q$ of hexaploid wheat. Genetics. 1963:48:469-82.

4. Faris JD, Gill BS. Genomic targeting and high-resolution mapping of the domestication gene Q in wheat. Genome. 2002;45:706-18.

5. Pourkheirandish M, Dai F, Sakuma S, Kanamori H, Distelfeld A, Willcox G, et al. On the origin of the non-brittle rachis trait of domesticated einkorn wheat. Front Plant Sci. 2018;8:1-10.

6. Zhao $Y$, Xie $P$, Guan $P$, Wang $Y, L i Y, Y u K$, et al. Btr $1-A$ induces grain shattering and affects spike morphology and yield-related traits in wheat. Plant Cell Physiol. 2019;60:1342-53.

7. Sharma JS, Running KLD, Xu SS, Zhang Q, Peters AR, Sharma S, et al. Genetic analysis of threshability and other spike traits in the evolution of cultivated emmer to fully domesticated durum wheat. Mol Genet Genomics. Springer Berlin Heidelberg. 2019;294:757-71.

8. Nave M, Avni R, Çakır E, Portnoy V, Sela H, Pourkheirandish M, et al. Wheat domestication in light of haplotype analyses of the Brittle rachis 1 genes (BTR1-A and BTR1-B). Plant Sci. 2019;285:193-9.

9. Sood S, Kuraparthy V, Bai G, Gill BS. The major threshability genes soft glume (sog) and tenacious glume (Tg), of diploid and polyploid wheat, trace their origin to independent mutations at non-orthologous loci. Theor Appl Genet. 2009;119:341-51.

10. Chuck G, Meeley R, Irish E, Sakai H, Hake S. The maize tasselseed4 microRNA controls sex determination and meristem cell fate by targeting Tasselseed6/ indeterminate spikelet1. Nat Genet. 2007:39:1517-21.

11. Sormacheva I, Golovnina K, Vavilova V, Kosuge K, Watanabe N, Blinov A, et al. $Q$ gene variability in wheat species with different spike morphology. Genet Resour Crop Evol. 2015;62:837-52.

12. Aukerman MJ, Sakai H. Regulation of flowering time and floral organ identity by a microRNA and its APETALA2-like target genes. Plant Cell. 2003; 15:2730-41.

13. Chen X. A microRNA as a translational repressor of APETALA2 in Arabidopsis flower development. Science. 2004;303:2022-5.

14. Jung J-H, Seo Y-H, Seo PJ, Reyes JL, Yun J, Chua N-H, et al. The GIGANTEAregulated microRNA172 mediates photoperiodic flowering independent of CONSTANS in Arabidopsis. Plant Cell. 2007:19:2736-48.

15. Chuck G, Meeley RB, Hake S. The control of maize spikelet meristem fate by the APETALA2-like gene indeterminate spikelet1. Genes Dev. 1998; 12:1145-54

16. Debernardi JM, Lin H, Chuck G, Faris JD, Dubcovsky J. microRNA172 plays a crucial role in wheat spike morphogenesis and grain threshability. Development. 2017;144:1966-75.

17. Greenwood JR, Finnegan EJ, Watanabe N, Trevaskis B, Swain SM. New alleles of the wheat domestication gene $Q$ reveal multiple roles in growth and reproductive development. Development. 2017:144:1959-65.

18. Liu P, Liu J, Dong H, Sun J. Functional regulation of Q by microRNA172 and transcriptional co-repressor TOPLESS in controlling bread wheat spikelet density. Plant Biotechnol J. 2018;16:495-506. 
19. Zhao K, Xiao J, Liu Y, Chen S, Yuan C, Cao A, et al. Rht23 (5Dq') likely encodes a $Q$ homeologue with pleiotropic effects on plant height and spike compactness. Theor Appl Genet Springer Berlin Heidelberg. 2018;131: 1825-34.

20. Jiang $Y$, Chen $Q$, Wang $Y$, Guo Z, Xu B, Zhu J, et al. Re-acquisition of the brittle rachis trait via a transposon insertion in domestication gene $\mathrm{Q}$ during wheat de-domestication. New Phytol. 2019;224(2):961-73.

21. Golovnina KA, Glushkov SA, Blinov AG, Mayorov VI, Adkison LR, Goncharov NP. Molecular phylogeny of the genus Triticum L. Plant Syst Evol. 2007;264: 195-216.

22. Goncharov NP, Golovnina KA, Kilian B, Glushkov S, Blinov A, Shumny VK. Evolutionary history of wheats - the main cereal of mankind. In: Dobretsoval N, editor. Biosphere Origin and Evolution; 2008. p. 407-19.

23. Nishikawa K, Furuta Y, Wada T. Genetic studies of ALPHA-amylase isozymes in wheat. III. Intraspecific variation in Aegilops squarrosa and birthplace of hexaploid wheat. Japanese J. Genet. 1980;55:325-36.

24. Jaaska V. NADP-dependent aromatic alcohol dehydrogenase in polyploid wheats and their diploid relatives. On the origin and phylogeny of polyploid wheats. Theor. Appl. Genet. 1978:53:209-17.

25. Jaaska V. Aspartate aminotransferase and alcohol dehydrogenase isoenzymes: intraspecific differentiation in Aegilops tauschii and the origin of the D genome polyploids in the wheat group. Plant Syst Evol. 1981;137: 259-73.

26. Dvorak J, Luo MC, Yang ZL, Zhang HB. The structure of the Aegilops tauschil genepool and the evolution of hexaploid wheat. Theor Appl Genet. 1998; 97:657-70.

27. Regel RE. Grain crops in Russia. Tr Prikl Bot Sel. 1922;13.

28. Chen QF, Yen C, Yang JL. Chromosome location of the gene for the hulled character in the Tibetan weedrace of common wheat. Genet Resour Crop Evol. 1998:45:407-10.

29. Dorofeev VF, Korovina ON. Kul'turnaya flora SSSR (Cultivated Flora of the Soviet Union). Leningrad, Kolos: Leningrad Branch; 1979.

30. Flaksberger KA. Pshenitsi- rod Triticum L. (Wheats- genus Triticum L.). Kul'turnaya flora SSSR. Khlebnie zlaki. Pshenitsa. (Cultivated Flora Sov. Union. Cereal. Wheat.). Gosudarstvennoe izdatel'stvo sovhoznoi i kolhoznoi literaturi. Moskva- Leningrad; 1935. p. 17-434.

31. Genaev MA, Komyshev EG, Smirnov NV, Kruchinina W, Goncharov NP Afonnikov DA. Morphometry of the wheat spike by analyzing $2 \mathrm{D}$ images. Agronomy. 2019;9:390

32. Katoh K, Misawa K, Kuma K, Miyata T. MAFFT: a novel method for rapid multiple sequence alignment based on fast Fourier transform. Nucleic Acids Res. 2002;30:3059-66.

33. Larsson A. Aliview: a fast and lightweight alignment viewer and editor for large datasets. Bioinformatics. 2014;30:3276-8.

34. Jukes TH, Cantor CR. Evolution of protein molecules. Mamm. Protein Metab. New York: Academic Press; 1969. p. 21-132.

35. Nguyen L-T, Schmidt HA, von Haeseler A, Minh BQ. IQ-TREE: a fast and effective stochastic algorithm for estimating maximum-likelihood phylogenies. Mol Biol Evol. 2015;32:268-74.

36. Hoang DT, Chernomor O, von Haeseler A, Quang Minh B, Sy Vinh L. UFBoot2: Improving the ultrafast bootstrap approximation. Mol Biol Evol. 2017:35:518-22.

37. Dai X, Zhuang Z, Zhao PX. PsRNATarget: a plant small RNA target analysis server (2017 release). Nucleic Acids Res Oxford University Press. 2018;46: W49-54.

\section{Publisher's Note}

Springer Nature remains neutral with regard to jurisdictional claims in published maps and institutional affiliations.

Ready to submit your research? Choose BMC and benefit from:
- fast, convenient online submission
- thorough peer review by experienced researchers in your field
- rapid publication on acceptance
- support for research data, including large and complex data types
- gold Open Access which fosters wider collaboration and increased citations
- maximum visibility for your research: over 100M website views per year
At BMC, research is always in progress.
Learn more biomedcentral.com/submissions

